\title{
Populus deltoides 'Australiano 129/60': variación axial de la densidad y desarrollo de un modelo predictivo de la densidad del árbol completo
}

\author{
Populus deltoides clone 'Australian 129-60': density axial variation and predictive model \\ for whole tree density
}

\author{
Gastón Diaz ${ }^{a}$, Silvia Monteoliva ${ }^{a *}$, Javier Álvarez ${ }^{b}$, Ezequiel Fernández Tschieder ${ }^{b}$

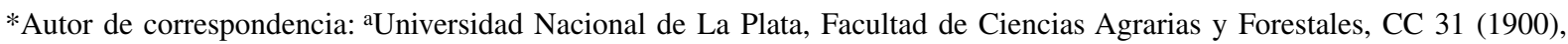 \\ La Plata, Argentina, tel.: 54-0221-4236616, fax: 54-0221-4252346, smonteoliva@yahoo.com.ar
}

bINTA EEA Delta del Paraná, Campana, Buenos Aires, Argentina.

\begin{abstract}
SUMMARY
The aims were to determine the axial variation pattern for wood density of Populus deltoides clone 'Australian 129-60' and to develop a predictive model for whole tree density from density at one sample height. Eleven ten-year-old trees from three diametric classes were sampled in Buenos Aires Province, Argentine. Disks were taken at seven sampling heights $(0.5 \mathrm{~m} ; 0.7 \mathrm{~m} ; 1.0 \mathrm{~m} ; 2.2 \mathrm{~m}$; $6.6 \mathrm{~m} ; 11.0 \mathrm{~m}$ and $15.4 \mathrm{~m})$. Density and weighted density were determined at $15 \%$ of moisture content. An analysis of variance was carried out for sampling heights and diametric classes. The relationship between whole tree density and predictive parameters, density at $1.0 \mathrm{~m}$ height and Diameter at breast height (DBH), was quantified using linear and non linear regression analyses. Clone mean density was $458 \pm 35 \mathrm{~kg} \mathrm{~m}^{-3}$ and weighted density was $470 \pm 14 \mathrm{~kg} \mathrm{~m}^{-3}$. Density axial variation shows significantly increasing values from the lower sampling height to the top. Density taken at breast height, approximately $(1.0 \mathrm{~m})$, underestimates $(11 \%)$ the weighted density of whole tree. It is feasible to produce high diameters poplars and to obtain major wood density at the same time. It is possible to fit models to estimate the mean weighted density of whole tree based on density at breast height approximately, with linear regression analysis.
\end{abstract}

Key words: density at $15 \% \mathrm{MC}$, axial variation, diametric classes, DBH-density relationship, prediction models.

\section{RESUMEN}

Los objetivos de este trabajo fueron determinar la variación axial de la densidad de la madera de Populus deltoides 'Australiano 129/60' y desarrollar un modelo predictivo de la densidad total del árbol a partir de la densidad a una única altura. Se voltearon 11 árboles de 10 años de tres clases diamétricas en Buenos Aires, Argentina. Se extrajeron muestras a siete alturas del fuste $(0,5 \mathrm{~m}$; $0,7 \mathrm{~m} ; 1,0 \mathrm{~m} ; 2,2 \mathrm{~m} ; 6,6 \mathrm{~m} ; 11,0 \mathrm{~m}$ y $15,4 \mathrm{~m})$. Se determinó la densidad normal media y media ponderada por volumen al $15 \%$ de contenido de humedad. Se realizaron ANOVAs entre alturas de muestreo y clases diamétricas. Se realizaron regresiones lineales y no lineales, simples y múltiples, para ajustar modelos de predicción de la densidad media ponderada del fuste, tomando como variables predictoras la densidad normal a $1 \mathrm{~m}$ de altura y el diámetro a la altura del pecho. La densidad media fue de $458 \pm 35$ $\mathrm{kg} \mathrm{m}^{-3}$ y la densidad ponderada de $470 \pm 14 \mathrm{~kg} \mathrm{~m}^{-3}$. La variación axial mostró un aumento significativo de los valores de densidad desde la base hacia el ápice. La densidad tomada a 1,0 m subestimó la densidad ponderada del árbol completo en un $11 \%$. Se verificó una mayor densidad en las clases diamétricas superiores. Se considera factible producir estos álamos con mayores diámetros y obtener al mismo tiempo mayor densidad. Es posible ajustar modelos de estimación de la densidad media ponderada a partir de una muestra tomada a una única altura y el DAP.

Palabras clave: densidad de referencia al 15\%, variación axial, clases diamétricas, relación DAP-densidad, modelos de predicción.

\section{INTRODUCCIÓN}

El delta del río Paraná abarca una superficie aproximada de $17.500 \mathrm{~km}^{2}$ en el NE de la provincia de Buenos Aires y extremo austral de Entre Ríos, entre los $32^{\circ} 5^{\prime}$ y $34^{\circ} 29^{\prime}$ $\mathrm{S}$ y $58^{\circ} 22^{\prime}$ y $60^{\circ} 45^{\prime} \mathrm{O}$ (Bonfils 1962). Constituye una excelente región para la producción de maderas blandas con fines industriales (Bonetto y Hurtado 1998). En la actualidad la forestación con salicáceas, de los géneros Populus spp. (álamos) y Salix spp. (sauces), representa la principal actividad productiva de la región (Borodowski y Suárez 2005) con aproximadamente 56.000 ha forestadas 
(SAGPyA 2001). Las plantaciones de álamo se concentran en el Delta Bonaerense, donde existen aproximadamente 10.000 ha bajo manejo intensivo, que en la mayoría de los casos se encuentran protegidas por un endicamiento perimetral (Borodowski y Suárez 2005). La madera obtenida se destina principalmente a aserrado y debobinado.

La densidad de la madera es una propiedad universalmente utilizada como índice de calidad en relación a sus usos dado que está correlacionada con la mayoría de las propiedades físico-mecánicas (Barnett y Jeronimidis 2003). Resulta de las dimensiones celulares, de la composición química y de las proporciones relativas de los tejidos constitutivos. Esta propiedad puede variar ampliamente dentro de un árbol, desde la médula hacia el exterior (en las diferentes edades) o axialmente desde la base del tronco hacia el ápice (Zobel y Talbert 1988). Conocer estas variaciones permitiría realizar muestreos de madera representativos del valor del árbol completo y reconocer diferencias de calidad en relación a los usos (Downes et al. 1997).

Los modelos de variación radial y axial de la densidad ya han sido estudiados en Populus spp. y en algunos otros géneros de latifoliadas de porosidad difusa (Eucalyptus spp., Acacia spp. y Salix spp.). Los resultados indicaron que la variación axial de la densidad es de menor magnitud que la variación radial (Wilkes 1988). En estos géneros, se manifiesta un aumento de la densidad desde la base al ápice (Downes et al. 1997, Igartúa et al. 2000, 2002, 2004, Monteoliva et al. 2002a). Asimismo, algunos autores describieron para el género Eucalyptus una reducción inicial de la densidad de la madera hasta el 10-25\% de la altura comercial y su aumento posterior (Bhat et al. 1990, Raymond y Muneri 2001, Clark 2001, Monteoliva et al. 2002b). Esta tendencia también es citada para diferentes clones del género Populus. Así fue demostrado para tres clones de Populus tremuloides Michx. por Yanchuk et al. (1983) en Canadá; también en ese país, Beaudoin et al. (1992) lo verificaron para 10 clones de 9 años de Populus $x$ canadensis Moench. Gutiérrez y Baonza (2001) publicaron igual tendencia en 25 clones de diferentes cruzamientos en España.

Para estimar la densidad del árbol completo a partir de un único punto de muestreo (por ejemplo, la altura del pecho, AP), debe hacerse una regresión entre todos los valores de densidad del árbol y la correspondiente a dicha altura (Zobel y Talbert 1988). Existen trabajos de este tipo para especies comerciales como Eucalyptus globulus Labill. (Raymond et al. 1998, Clark 2001, Igartúa et al. 2003), E. grandis Hill (ex Maiden) (Busnardo et al. 1985, 1987, Bhat et al. 1990, Clark 2001), E. nitens Deane et Maiden (Raymond et al. 1998), Salix babylonica var. sacramenta H. (Villegas y Marlats 2005) donde se demuestra y analiza la precisión que brindan las valoraciones obtenidas a la AP como predictoras de la densidad. Para álamo, solamente el trabajo de Beaudoin et al. (1992), realizado en Canadá, involucra este tipo de ajuste. Estos autores encontraron una correlación alta y positiva entre la densidad completa del árbol tomada como promedio de cuatro alturas en 10 clones de Populus $x$ canadensis y la densidad obtenida con muestra extraída con taladro de incrementos a la AP.

A partir de los antecedentes expuestos, los objetivos de este trabajo son determinar las tendencias en la variación axial de la densidad de la madera de Populus deltoides 'Australiano 129/60' y desarrollar un modelo predictivo de la densidad total del árbol a partir de la densidad a una única altura y del DAP.

\section{MÉTODOS}

El estudio se llevó a cabo en la zona del Delta Bonaerense localmente denominada núcleo forestal. Esta área se ubica dentro de la Unidad IV "Pajonales y bosques de las islas deltaicas" (Kandus et al. 2006) del Bajo Delta Bonaerense (Bonfils 1962).

Se seleccionaron dos parcelas de $1.500 \mathrm{~m}^{2}\left(34^{\circ} 08^{\prime}\right.$ $\left.\mathrm{S}, 58^{\circ} 43^{\prime} \mathrm{O}\right)$ en una plantación de Populus deltoides 'Australiano 129/60' de 10 años de edad protegida por un dique perimetral. La plantación había sido realizada con estacas de aproximadamente $80 \mathrm{~cm}$ de largo utilizando un distanciamiento de 5 x 3 m (667 árboles ha-1) y al quinto año el productor realizó un raleo selectivo de los árboles de menores diámetros o defectuosos en el cual se removieron aproximadamente el $30 \%$ de los individuos, razón por la cual actualmente la densidad de la plantación es de 407 y 413 árboles ha ${ }^{-1}$.

En cada parcela se midió el diámetro a la altura del pecho (DAP) de todos los individuos y se definieron clases diamétricas con intervalos de $5 \mathrm{~cm}$. En total se voltearon 11 individuos distribuidos entre las tres clases diamétricas de mayor frecuencia, a los cuales se midió la altura total (cuadro 1).

Cuadro 1. Dendrometría de los árboles muestreados. Dendrometric of sample trees.

\begin{tabular}{cccccc}
\hline Árbol & Parcela & $\begin{array}{c}\text { DAP } \\
(\mathrm{cm})\end{array}$ & $\begin{array}{c}\mathrm{Ht} \\
(\mathrm{m})\end{array}$ & $\begin{array}{c}\text { Volumen } \\
\left(\mathrm{m}^{3}\right)\end{array}$ & $\begin{array}{c}\text { Clase } \\
\text { diamétrica }\end{array}$ \\
\hline 1 & A & 21,8 & 22,1 & 0,256 & II \\
2 & B & 22,2 & 23,6 & 0,237 & $(20$ a 25 \\
3 & B & 23,6 & 22,4 & 0,275 & $\mathrm{~cm})$ \\
4 & A & 23,8 & 22,8 & 0,287 & \\
\hline 5 & B & 25,4 & 23,5 & 0,330 & III \\
6 & B & 26,8 & 23,3 & 0,345 & $(25$ a 30 \\
7 & A & 27,0 & 24,3 & 0,375 & $\mathrm{~cm})$ \\
8 & B & 27,1 & 24,2 & 0,367 & \\
\hline 9 & B & 31,0 & 23,8 & 0,496 & IV \\
10 & A & 31,3 & 25,5 & 0,497 & $(30$ a 35 \\
11 & B & 35,9 & 24,2 & 0,651 & $\mathrm{~cm})$ \\
\hline
\end{tabular}

DAP: diámetro a la altura del pecho, Ht: altura total del árbol. 
De cada uno de los árboles fueron extraídas secciones de troza a siete alturas, a razón de una pieza por altura (0,5 m;0,7 m; 1,0 m;2,2 m; 6,6 m; 11,0 m y 15,4 m). Las alturas de muestreo responden al tipo de aprovechamiento en trozas de 2,2 m de largo. Las secciones para la determinación de densidad fueron extraídas de la porción inferior de cada troza. Además se incluyeron dos alturas intermedias en la primera troza como posibles alturas predictoras de la densidad completa del árbol. Así, la primera troza contiene cuatro alturas de muestreo, la segunda, cuarta, sexta y novena trozas una muestra cada una. La muestra correspondiente a la altura del pecho $(1,3 \mathrm{~m})$ no estuvo disponible. La última altura muestreada $(15,4 \mathrm{~m})$ representó entre el 60 y $70 \%$ de la altura total del árbol.

Las muestras fueron acondicionadas previamente a la determinación de densidad. A cada sección de troza se le retiró la corteza y se seccionó en dos mitades utilizadas como repeticiones para la misma altura. La densidad de cada altura se calculó como el promedio simple de las dos repeticiones (medias secciones) y la densidad completa del árbol fue ponderada por el volumen del fuste. Se calculó el volumen de las trozas comprendidas entre alturas de muestreo, teniendo como altura inicial de la primera troza 0,3 m. Para la cubicación se utilizó la ecuación de Smalian. Como instrumental de medición de la densidad se utilizó una balanza digital con una capacidad máxima de $15 \mathrm{~kg}$ y una precisión de 0,2 g. Se registró el peso inicial, el volumen por inmersión total de la pieza en agua, y el peso seco en estufa a $103^{\circ} \mathrm{C} \pm 2^{\circ} \mathrm{C}$, pesado inmediatamente después de que la pieza alcanzara un equilibrio térmico con el ambiente. El contenido de humedad $(\mathrm{CH})$ promedio al que llegaron las muestras fue de $20 \%$, por lo que fue corregido según norma EN 384 (Europäisches Komitee für Normung 1996) para llevarla al 15\% (humedad de equilibrio higroscópico para la zona de La Plata, Buenos Aires). Se calculó la densidad de cada altura referida a un $15 \%$ de contenido de humedad (Coronel 1994) según la ecuación 1. Para el árbol completo, la densidad normal ponderada según el volumen de las trozas se calculó con la ecuación 2.

$$
D n \%_{i}=\frac{P \%_{i}}{V \%_{i}}
$$

Donde,

$D n \%_{i}$ : densidad normal con un contenido de humedad de $i \%$.

$P \%_{i} \quad$ : peso con un contenido de humedad de $i \%$.

$V \%_{i}$ : volumen con un contenido de humedad de $i \%$.

$$
\operatorname{DnpT} \%_{i}=\frac{\sum D n j \%_{i} \times V j}{\sum V j}
$$

Donde,

DnpT $_{i}$ : densidad normal ponderada del árbol completo con un contenido de humedad $i \%$.
$D n j \%_{i}$ : densidad normal a la altura $j$ con un contenido de humedad $i \%$.

Vj $\quad$ : volumen de la troza que contiene la altura $j$.

Se realizó un análisis descriptivo y un análisis de la varianza (ANDEVA) utilizando un modelo factorial para las variables altura de muestreo y clase diamétrica. Para la comparación de medias se utilizó la prueba de Tukey con un nivel de significancia del $95 \%$. Se realizó una matriz de correlación lineal entre el diámetro de los árboles, la densidad obtenida a un metro $\left(\mathrm{D}_{1 \mathrm{~m}}\right)$ y la densidad media del árbol ponderada por volumen (DnpT). Se ajustaron modelos de regresión lineal y no lineal, simples y múltiples, por mínimos cuadrados, para predecir la densidad media del árbol ponderada por volumen, tomando a la densidad a $1 \mathrm{~m}$ de altura y el diámetro a la altura de pecho como variables independientes. Se verificaron los supuestos de homocedasticidad y normalidad. Las correlaciones entre variables independientes fueron no significativas.

\section{RESULTADOS}

Análisis de la varianza y patrón de variación axial. La densidad normal a las distintas alturas de muestreo varió entre $412 \mathrm{~kg} \mathrm{~m}^{-3}$ y $547 \mathrm{~kg} \mathrm{~m}^{-3}$, siendo la densidad media por árbol expresada como promedio simple de las siete alturas de muestreo de $458 \pm 13,5 \mathrm{~kg} \mathrm{~m}^{-3}$ y la densidad del árbol completo ponderada por volumen de $470 \pm 14,2 \mathrm{~kg}$ $\mathrm{m}^{-3}$. Se observó un leve descenso de los valores medios de densidad en las primeras alturas junto con un pronunciado incremento en las alturas superiores (cuadro 2).

La interacción entre la altura y la clase diamétrica no resultó significativa según el ANDEVA $(P=0,570)$, aunque estas variables resultaron fuentes significativas de variación individualmente. El patrón de variación axial hallado mostró un ascenso de los valores de densidad desde la base hacia el ápice (figura 1). Las cuatro primeras alturas de muestreo $(0,5 \mathrm{~m}$ a $2,2 \mathrm{~m})$ no se diferenciaron significativamente $(P>0,05)$, es decir, que la primera troza comercial puede considerarse de densidad homogénea en su longitud. Las diferencias se presentaron entre los 2,2 $\mathrm{m}$ y los 6,6 m $(P<0,05)$, y entre éstos y los $11 \mathrm{~m}(P<0,05)$. No hubo diferencias entre los $11 \mathrm{~m}$ y los 15,4 m (cuadro 2, prueba de Tukey).

Para la variable diámetro se observó que la clase diamétrica menor (clase II), con una densidad promedio de $445 \mathrm{~kg} \mathrm{~m}^{-3}$, resultó significativamente inferior $(P<0,05)$ a las otras dos clases diamétricas (clases III: $464 \mathrm{~kg} \mathrm{~m}^{-3}$ y IV: $466 \mathrm{~kg} \mathrm{~m}^{-3}$ ), las cuales a su vez no se diferenciaron entre sí.

Correlaciones y regresiones. La correlación entre la densidad media del árbol, como promedio simple de las siete alturas (DMA), y la densidad obtenida a 1 metro $\left(D_{1 \mathrm{~m}}\right)$ fue de 
Cuadro 2. Densidad $\left(\mathrm{kg} \mathrm{m}^{-3}\right)$ al $15 \%$ de contenido de humedad por árbol (promedio simple y ponderado por volumen) y por altura de muestreo.

Density $\left(\mathrm{kg} \mathrm{m}^{-3}\right)$ at $15 \% \mathrm{MC}$ for tree and at sample heights.

\begin{tabular}{cccccccccc}
\hline & \multicolumn{10}{c}{ Altura de muestreo (m) } \\
\cline { 2 - 9 } Árbol* & 0,5 & 0,7 & 1 & 2,2 & 6,6 & 11 & 15,4 & $\begin{array}{c}\text { Densidad } \\
\text { por árbol }\end{array}$ & $\begin{array}{c}\text { Densidad } \\
\text { ponderada }\end{array}$ \\
\hline 1 (II) & 447 & 420 & 420 & 430 & 475 & 460 & 463 & 445 & 458 \\
3 (II) & 424 & 419 & 417 & 428 & 447 & 456 & 493 & 441 & 449 \\
2 (II) & 436 & 423 & 414 & 415 & 456 & 503 & 463 & 444 & 457 \\
5 (II) & 427 & 420 & 422 & 439 & 453 & 478 & 514 & 450 & 461 \\
4 (III) & 427 & 412 & 422 & 436 & 455 & 501 & 495 & 449 & 464 \\
9 (III) & 452 & 448 & 440 & 450 & 488 & 498 & 547 & 475 & 486 \\
6 (III) & 460 & 459 & 434 & 447 & 462 & 493 & 527 & 469 & 474 \\
7 (III) & 453 & 442 & 436 & 443 & 480 & 490 & 514 & 465 & 477 \\
8 (IV) & 430 & 420 & 416 & 425 & 463 & 498 & 504 & 451 & 466 \\
10 (IV) & 454 & 440 & 453 & 447 & 484 & 543 & 421 & 477 & 493 \\
11 (IV) & 442 & 434 & 424 & 443 & 485 & 530 & 533 & 470 \\
\hline Densidad por altura** & $441 \mathrm{a}$ & $431 \mathrm{a}$ & $427 \mathrm{a}$ & $437 \mathrm{a}$ & $468 \mathrm{~b}$ & $495 \mathrm{c}$ & $506 \mathrm{c}$ & 487 \\
\hline
\end{tabular}

*En paréntesis la clase diamétrica. **Letras iguales no difieren significativamente $(P<0,05)$.

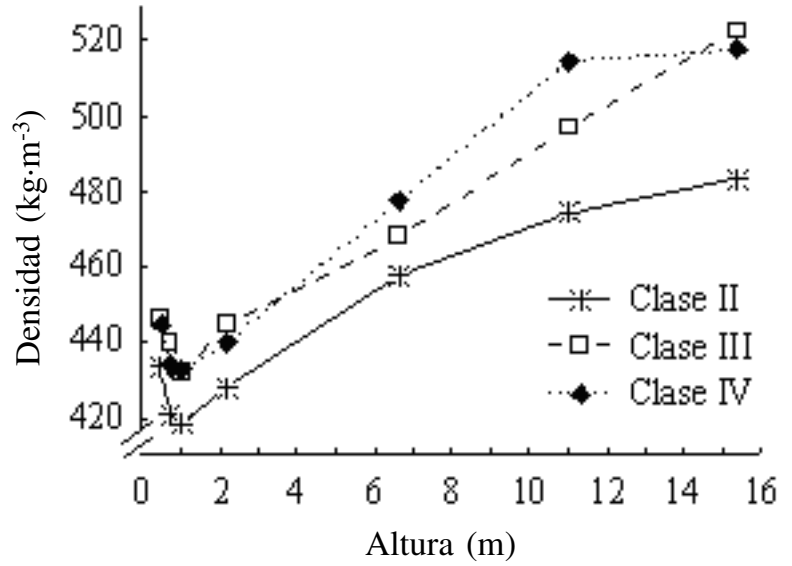

Figura 1. Densidad según las alturas de muestreo y clases diamétricas.

Density at sample heights and for diametric classes.

$0,87(P<0,05)$, mientras que con la densidad ponderada total (DnpT) fue de 0,82 $(P<0,05)$. Las correlaciones del diámetro de los árboles (DAP) con la DMA, con la DnpT y con la $\mathrm{D}_{1 \mathrm{~m}}$ fueron de $0,68(P<0,05), 0,77(P<0,05)$ y de $0,42 \mathrm{~ns}(P>0,05)$, respectivamente.

La función lineal simple indicada en la ecuación [2] relaciona la DnpT y la $D_{1 \mathrm{~m}}$, con un ajuste bajo $\left(\mathrm{R}^{2}=0,65\right.$; $P<0,05)$. La figura 2 presenta gráficamente el modelo y la figura 3 el análisis de los residuos.

$$
\text { DnpT }=57,2+0,966 \mathrm{D}_{1 \mathrm{~m}}
$$

La densidad evaluada a la altura de un metro subestimó en un $11 \%$ la densidad del árbol ponderada por volumen. Los errores de este modelo fueron inferiores al $5 \%$.

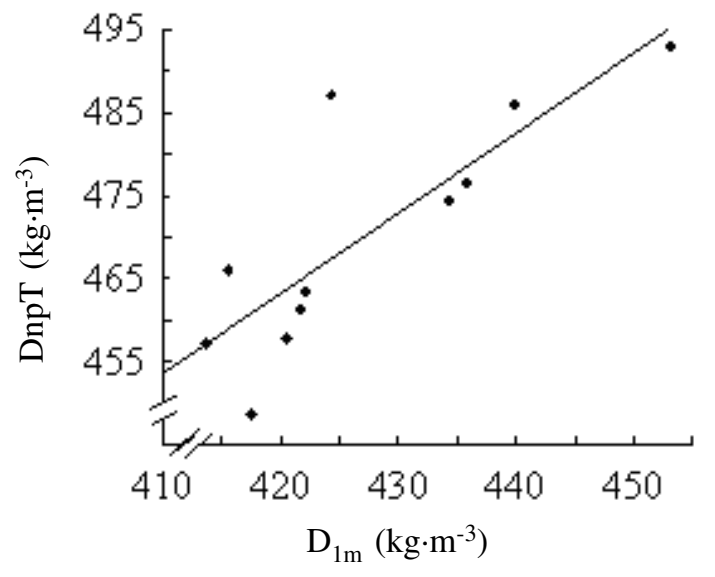

Figura 2. Densidad ponderada del árbol (DnpT) en función de la densidad a $1 \mathrm{~m}(\mathrm{D} 1 \mathrm{~m})$.

Mean density (DnpT) by density at $1 \mathrm{~m}(\mathrm{D} 1 \mathrm{~m})$.

La ecuación [3] presenta un modelo no lineal que resultó no significativo $(P>0,05)$. No se logró un mejor ajuste con la ecuación polinomial [3] en relación a la ecuación simple [2].

$$
\text { DnpT }=-1843,4+9,76 \mathrm{D}_{1 \mathrm{~m}}-0,0101 \mathrm{D}_{1 \mathrm{~m}}{ }^{2}
$$

La función lineal múltiple indicada en la ecuación [4] relaciona la DnpT con la $\mathrm{D}_{1 \mathrm{~m}}$ y el DAP, con un buen ajuste $\left(\mathrm{R}^{2}=0,92 ; P<0,05\right)$. La figura 3 presenta gráficamente el análisis de los residuos.

$$
\text { DnpT }=107,66-0,736 D_{1 m}+178,79 \text { DAP }
$$




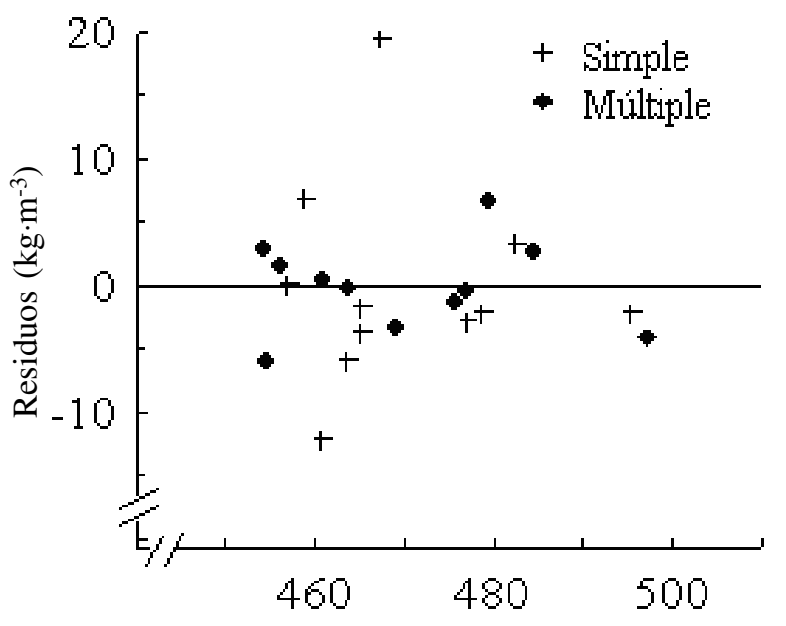

Valores predichos DnpT $\left(\mathrm{kg} \cdot \mathrm{m}^{-3}\right)$

Figura 3. Gráfico de residuos para la regresión lineal simple y múltiple. multiple regressions.

Residual graphics for linear simple regression and for

Los residuos de la regresión múltiple (figura 3) tienen menos magnitud que los presentados en la regresión simple. La evaluación de los modelos según el $\mathrm{R}^{2}$ ajustado, destaca también a la ecuación lineal múltiple [4] frente a la simple [2].

\section{DISCUSIÓN}

El valor medio de densidad de todos los árboles muestreados fue de $458 \mathrm{~kg} \mathrm{~m}^{-3}$, lo cual ubica a este clon entre los álamos de mayor densidad implantados en Argentina para la edad que representan (10 años) (Senisterra et al. 2005). La densidad al $15 \%$ de contenido de humedad reportada para este clon $\left(504 \mathrm{~kg} \mathrm{~m}^{-3}\right)$ es un poco más alta (Petray 1998), aunque en esta referencia no se indica la edad de los árboles tomados como muestra. Es conocida la influencia de la edad en las propiedades de la madera: a mayor edad la densidad media aumenta (Barnet y Jeronimidis 2003). Análisis de densidad básica realizados por Senisterra et al. (2005) en clones de nueve años en Provincia de Santa Fe, Argentina, destacan a cinco clones de $P$. deltoides entre los de mayor densidad, frente a siete clones de $P$. $x$ canadensis.

El patrón de variación axial hallado en el presente estudio mostró un aumento de los valores de densidad desde la base del árbol hacia el ápice. En España, Gutiérrez y Baonza (2001), en un estudio de propiedades físicas sobre 176 árboles de 25 clones de álamos con edades comprendidas entre 14 y 17 años, hallaron la misma tendencia que en este estudio. Reportaron diferencias significativas de densidad básica entre cuatro alturas de muestreo en el fuste, tomados a partir de la altura del pecho. Contrariamente, un patrón diferente fue publicado para 10 clones de $P$. $x$ canadensis con nueve años de edad procedentes de una plantación en el sureste de Canadá, en la cual se muestrearon cuatro alturas de 28 árboles. En este trabajo, las diferencias fueron significativas entre las cuatro alturas testeadas. Los menores valores de densidad se presentaron entre $1 \mathrm{~m}$ y $3 \mathrm{~m}$, siendo en la base y en la altura máxima muestreada $(4,5 \mathrm{~m})$, de mayor magnitud (Beaudoin et al. 1992). También en Canadá, pero con tres clones de $P$. tremuloides de 40 a 47 años de edad, Yanchuk et al. (1983) encontraron un patrón de variación similar al mencionado por Beaudoin et al. (1992). Estos autores registraron una reducción significativa del peso específico de la madera entre los $2 \mathrm{~m}$ y los $8 \mathrm{~m}$ de altura, en un estudio realizado con cuatro árboles por clon y cinco alturas de muestreo en el fuste.

Para condiciones de crecimiento similares a las del material analizado en este trabajo, es esperable que el clon 'Australiano 129/60' presente densidades significativamente mayores en las clases diamétricas medias y superiores. Este resultado es promisorio ante la decisión de incorporar alguna práctica silvicultural que tienda a aumentar la tasa de crecimiento (fertilización, irrigación, raleo), porque no perjudica la densidad de la madera producida.

Existe una correlación entre la densidad tomada a un metro en el fuste y la densidad media y media ponderada del árbol para el clon 'Australiano 129/60'. Coincidentemente con este resultado, Beaudoin et al. (1992) publicaron una correlación significativa de $\mathrm{r}=0,96(P<0,05)$ entre la densidad completa del árbol ponderada por volumen y la densidad a la altura del pecho determinada sobre una muestra de taladro de incremento, para 10 clones $P$. $x$ canadensis de nueve años.

En general, los clones de $P$. deltoides presentan un rápido crecimiento, por lo tanto es importante conocer la naturaleza de la relación de dicho crecimiento con la densidad de la madera. Beaudoin et al. (1992) determinaron una correlación negativa $(\mathrm{r}=-0,46 ; P<0,05)$, mientras que en Estados Unidos se han publicado trabajos que reportan la independencia de estas variables en diferentes clones de álamos (Marton et al. 1968, Holt y Murphey 1978).

La correlación entre DAP y densidad media (DMA) y media ponderada del árbol (DnpT) fueron positivas y significativas en nuestro estudio, por lo que se espera que a mayor crecimiento la densidad promedio aumente. En Argentina, otros trabajos indican que la densidad de la madera puede ser independiente de la velocidad de crecimiento en álamos. Así, Senisterra et al. (2005) determinaron que no hubo correlación significativa entre DAP y densidad básica (medida a la altura de pecho) para 12 clones de álamos (de $P$. deltoides y $P$. $x$ canadensis) de nueve años implantados en la provincia de Santa Fe. Bonavía y Piussan (1989) obtienen el mismo resultado en un estudio sobre 10 clones en el Delta de Paraná. Ambos trabajos se basaron en datos de crecimiento y densidad determinados sobre ensayos comparativos de clones, estableciendo de este 
modo que la selección por crecimiento de determinado clon no tendrá una influencia directa sobre la densidad y en consecuencia en el rendimiento de madera. No existen trabajos publicados que muestren la relación entre DAP y densidad para clones de álamos de plantaciones comerciales o ensayos puros de Argentina.

El patrón de variación axial del clon 'Australiano 129/60' logró ser modelado por regresión lineal. Se probaron dos ecuaciones de ajuste para predecir la densidad media ponderada del árbol, prefiriéndose la ecuación lineal múltiple DnpT $=107,66-0,736 \mathrm{D}_{1 \mathrm{~m}}+178,79$ DAP por ser la de mejor $\mathrm{R}^{2}$ ajustado. La importancia de esta ecuación radica en la vinculación de dos variables de rápida determinación como son el DAP y la densidad a una única altura posible de determinarse por medios no destructivos. Estos modelos deberán ser validados con un mayor número de árboles, incluyendo además plantaciones de otros sitios de la región del Delta del Paraná. La única referencia bibliográfica hallada para el género Populus que presenta modelos de predicción fue la de Beaudoin et al. (1992). El modelo presentado por estos autores predice la densidad ponderada completa del árbol a partir de la densidad obtenida a la altura de pecho (AP) sobre una muestra de taladro de incremento. La ecuación se presenta en el cuadro 3 junto con otras ecuaciones publicadas en la bibliografía para géneros comerciales de angiospermas.

Si bien un solo trabajo se refiere al género Populus, se muestra que varios autores han trabajado en otras especies de porosidad difusa obteniendo resultados que concuerdan con el presente estudio. Los distintos autores han encontrado buenos ajustes entre la densidad media del árbol (obtenida como promedio simple o ponderado) y la densidad medida a la AP. Para el género Eucalyptus, otros autores hallaron mejores predicciones para la densidad media a partir del 25 a $50 \%$ de la altura comercial del árbol (Busnardo et al. 1985, 1987, Dean et al. 1990), aunque dichas alturas son poco prácticas para la determinación de la densidad en árboles en pie.

Diversas referencias se hallaron para determinaciones a partir de alturas fijas (entre 0,7 y $1,5 \mathrm{~m}$ ) como posibles puntos de extracción de las muestras (Downes et al. 1997, Raymond et al. 1998, Raymond y Muneri 2001). En estos trabajos, como el presente, se priorizó que la "altura predictora" sea de fácil acceso, para evitar un muestreo destructivo en la determinación del valor medio del árbol.

Es necesario realizar otras determinaciones de densidad de este genotipo en otros sitios de implantación y a otras edades. Asimismo, sería necesario realizar la validación del modelo con valores provenientes de otros árboles de este clon, con igual edad y condiciones de crecimiento.

El uso de las ecuaciones presentadas debe restringirse a las mismas condiciones planteadas en este estudio, ya que la utilización indiscriminada de este tipo de modelos puede introducir errores en las estimaciones que comprometan decisiones posteriores.

\section{CONCLUSIONES}

Se verifica un patrón de variación axial en la densidad normal del fuste que muestra un aumento de los valores desde la base hacia el ápice. La densidad tomada aproximadamente a la altura del pecho subestima la densidad ponderada del árbol completo en un $11 \%$.

La correlación entre la densidad media del árbol y el DAP sugiere que es posible producir álamos del clon 'Australiano 129/60' de mayores diámetros, con las técnicas silvícolas adecuadas, y obtener al mismo tiempo mayor densidad en la madera producida para condiciones de crecimiento similares a las de este estudio.

Es posible ajustar, con técnicas de regresión lineal, modelos de estimación de la densidad media ponderada del árbol completo a partir de una muestra tomada aproximadamente a la altura del pecho y su valor de DAP. El modelo propuesto es: DnpT $=107,66-0,736$ $\mathrm{D}_{1 \mathrm{~m}}+178,79$ DAP. Esta ecuación permite la estimación de la densidad media ponderada del árbol completo a partir de dos variables de rápida determinación, evitando así un muestreo destructivo.

Cuadro 3. Modelos predictivos de la densidad media del árbol (y) basados en la determinación a la altura del pecho ( $\mathrm{x}=\mathrm{AP}$ ). Predictive models for density of the whole tree $(y)$, based on density determined at breast height $(x=B H)$.

\begin{tabular}{llllll}
\hline Autor & Género/especie & $\mathrm{N}$ & Edad (años) & Ecuación & $\mathrm{r}$ o R \\
\hline Beaudoin et al.(1992) & Populus sp. & 28 & 9 & $\mathrm{y}=52,76+0,86 \mathrm{x}$ & $\mathrm{r}=0,93^{*}$ \\
Busnardo et al. $(1987)$ & Eucalyptus grandis & 59 & $6-7$ & $\mathrm{y}=0,208+0,5268 \times$ & $\mathrm{R}^{2}=0,53 *$ \\
Bhat et al. (1990) & E. grandis & 25 & 5 & $\mathrm{y}=0,1173+0,750 \mathrm{x}$ & $\mathrm{R}^{2}=0,90^{*}$ \\
Igartúa et al. (2003) & E. globulus & 20 & 35 & $\mathrm{y}=0,1482+0,8054 \mathrm{x}$ & $\mathrm{R}^{2}=0,80^{*}$ \\
Villegas y Marlats (2005) & Salix sp. & 32 & $8-13$ & $\mathrm{y}=135,4+0,6950 \times$ & $\mathrm{R}^{2}=0,80^{*}$ \\
\hline
\end{tabular}

$\mathrm{N}$ : número de árboles, $\mathrm{r}$ : coeficiente de regresión, $\mathrm{R}^{2}$ : coeficiente de determinación, $* P<0,05$. 


\section{REFERENCIAS}

Barnett JR, G Jeronimidis. 2003. Wood Quality and its biological basis. Boca Ratón, USA. CRC Press. 226 p.

Beaudoin M, RE Hernandez, A Koubaa, J Poliquin. 1992. Interclonal, intraclonal and within-tree variation in wood density of poplar hybrid clones. Wood Fiber Sci. 24(2): 147-153.

Bhat KM, KV Bhat, TK Dhamoradan. 1990. Wood density and fibre length of Eucalyptus grandis grown in Kerala, India. Wood Fiber Sci. 22(1): 54-61.

Bonavía E, CM Piussan. 1989. Evaluación con fines papeleros de diez clones de álamo en ensayos del INTA-Delta del Paraná. $25^{\circ}$ Congreso Técnico sobre Celulosa y Papel, ATIPCA, Argentina. p. 265-277.

Bonetto AA, S Hurtado. 1998. Región 1. Cuenca del Plata. In Canevari P, DE Blanco, EH Bucher, G Castro, I Davidson eds. Los humedales de la Argentina: clasificación, situación actual, conservación y legislación. Buenos Aires, Argentina. Wetland International Publ. 46 (2da Edición). p. 31-72.

Bonfils CG. 1962. Los suelos del Delta del Río Paraná. Factores generadores, clasificación y uso. Revista de Investigaciones Agrícolas 16: 257-370.

Borodowski ED, RO Suárez. 2005. Caracterización forestal de la región del Delta del Paraná. Núcleo Extensión Forestal Delta, Proyecto Forestal de Desarrollo, Secretaría de Agricultura, Ganadería, Pesca y Alimentación, Argentina. 8 p.

Busnardo CA, JV Gonzaga, CEB Foelkel, C Dias, S Menochelli. 1985. Em busca da qualidade ideal da madeira do eucalipto para produção de celulosa III. A importancia da altura de amostragem para avaliação da densidade básica media da arvore. San Pablo, Brasil. III Congreso Latinoamericano de Celulosa e Papel. p. 55-72.

Busnardo CA, JV Gonzaga, CEB Foelkel, S Menochelli. 1987. Em busca da qualidade ideal da madeira do eucalipto para produção de celulosa IV. Altura ideal de amostragem para avaliação da densidade basica media para arvore de Eucalyptus grandis. XX Congreso Anual da ABCP- Semana do Papel, San Pablo, Brasil. p. 17-33.

Clark N. 2001. Longitudinal density variation in irrigated hardwoods. Appita Journal 54(1): 49-53.

Coronel EO. 1994. Fundamentos de las propiedades físicas y mecánicas de las maderas. Aspectos teóricos y prácticos para la determinación de las propiedades y sus aplicaciones. Instituto de Tecnología de la Madera. Serie de publicaciones 9.404. Santiago del Estero, Argentina. Editorial El Liberal. 187 p.

Dean GH, J French, WN Tibbits. 1990. Variation in pulp and papermaking characteristics in a field trial of Eucalyptus globulus. 44 ${ }^{\text {th }}$ Annual General Conference Appita, Rotorua, New Zealand. p. 1-33.

Downes GM, I Hudson, C Raymond, A Dean, A Micheli, L Schimlek, R Evans, A Muneri. 1997. Sampling Eucalyptus for wood and fibre properties. Australia. CSIRO Publishing. $132 \mathrm{p}$.

Europäisches Komitee für Normung. 1996. DIN EN 384, Bauholz für tragende Zwecke, Bestimmung charakteristischer Festigkeits-, Steifigkeits- und Rohdichtewerte. Beuth Verlag, Berlin.
Gutiérrez Oliva A, MV Baonza Merino. 2001. Propiedades físicas de la madera de diferentes clones de chopo. I Simposio del Chopo, Zamora, España. p. 461-468.

Holt DH, WK Murphey. 1978. Properties of hybrid poplar juvenile wood affected by silvicultural treatments. Wood Science 10(4): 198-203.

Igartúa D, SM Rivera, MG Monterrubianesi, S Monteoliva, S Farina, S Carranza, M S Villegas. 2000. Calidad del leño en Eucalyptus globulus ssp. globulus. I- Variación de la densidad básica y el largo de fibras en una estación del sudeste de la Provincia de Buenos Aires, Argentina. I Congreso Iberoamericano de Investigación en Celulosa y Papel (CIADICYP). Misiones, Argentina. 8 p.

Igartúa DV, S Monteoliva, MG Monterrubianesi, MS Villegas. 2002. Calidad del leño en Eucalyptus globulus ssp. globulus. Variación de la densidad básica y la longitud de fibras en Lobería, Provincia de Buenos Aires, Argentina. Revista de la Facultad de Agronomía, La Plata 105(1): 35-45.

Igartúa DV, S Monteoliva, MG Monterrubianesi, MS Villegas. 2003. Basic density and fibre length at breast height of Eucalyptus globulus ssp. globulus for parameter prediction of the whole tree. IAWA Journal 24(2): 173-184.

Igartúa DV, S Monteoliva, C Núñez. 2004. Densidad básica, longitud de fibras y composición química de la madera de Eucalyptus globulus en el sudeste de la Provincia de Buenos Aires, Argentina. III Congreso Iberoamericano de Investigación en Celulosa y Papel (CIADICYP). Córdoba, España. 8 p.

Kandus P, RD Quintana, RF Bó. 2006. Patrones de paisaje y biodiversidad del Bajo Delta del Río Paraná. Mapa de ambientes. Buenos Aires, Argentina. Pablo Casamajor Ediciones. 48 p.

Marton R, GR Stairs, EJ Schreiner. 1968. Influence of growth rate and clonal effects on wood anatomy and pulping properties of hybrid poplars. Tappi 51(5): 230-235.

Monteoliva S, MG Senisterra, J Marquina, R Marlats, MS Villegas. 2002a. Estudio de la variación de la densidad básica en siete clones de Salix. Revista Facultad Agronomía La Plata 105(1): 29-34.

Monteoliva S, C Núñez, D Igartúa. 2002b. Densidad básica, longitud de fibras y composición química de una plantación de Eucalyptus globulus de la Provincia de Buenos Aires, Argentina. II Congreso Iberoamericano de Investigación en Celulosa y Papel (CIADICYP). San Pablo, Brasil. 8 p.

Petray EM. 1998. Aptitudes tecnológicas de distintos clones de Salix (sauces) y Populus (álamos), principales características físico-mecánicas. SAGPyA Forestal № 5: 13-17.

Raymond CA, A Muneri, AC Macdonald. 1998. Nondestructive sampling for basic density in Eucalyptus globulus and Eucalyptus nitens. Appita Journal 51(3): 224-227.

Raymond CA, A Muneri. 2001. Nondestructive sampling of Eucalyptus globulus and E. nitens for wood properties. I. Basic density. Wood Sci. Tech. 35: 27-39.

Secretaría de Agricultura, Ganadería, Pesca y Alimentación (SAGPyA). 2001. Inventario nacional de plantaciones forestales. SAGPyA. 63 p.

Senisterra G, MG Ducid, RM Marlats. 2005. Variación de la densidad básica de la madera en clones del género Populus. $3^{\mathrm{er}}$ Congreso Forestal Argentino y Latinoamericano. Corrientes, Argentina. 8 p. 
Villegas MS, RM Marlats. 2005. Altura de extracción de la muestra para evaluación de densidad básica y blancura en madera de Salix sp. Bosque 26(3): 121-132.

Yanchuk AD, BP Dancik, MM Micko. 1983. Intraclonal variation in wood density of trembling aspen in Alberta. Wood and Fiber Science 15(4): 387-394.

Wilkes J. 1988. Variation in wood anatomy within species of Eucalyptus. IAWA Bull. n.s. 9(1): 13-23.
Zobel B, J Talbert. 1988. Técnicas de mejoramiento genético de árboles forestales. Buenos Aires, Argentina. Limusa. $545 \mathrm{p}$.

Recibido: 09.06.09

Aceptado: 09.10.09 